\title{
A 48-Channel Receive Array Coil for Mesoscopic Diffusion-Weighted MRI of Human ex vivo Brain Imaging on the $3 \mathrm{~T}$ Connectome Scanner
}

\author{
Alina Scholz ${ }^{\mathrm{a}, *}$, Robin Etzel ${ }^{\mathrm{a}}$, Markus W May ${ }^{\mathrm{a}}$, Mirsad Mahmutovic ${ }^{\mathrm{a}}$, Qiyuan Tian ${ }^{\mathrm{b}, \mathrm{c}}$, Gabriel \\ Ramos-Llordén $^{\mathrm{b}, \mathrm{c}}$, Berkin Bilgiç, ${ }^{\mathrm{b}, \mathrm{c}, \mathrm{d}}$, Thomas Witzel ${ }^{\mathrm{b}, \mathrm{c}}$, Jason P Stockmann ${ }^{\mathrm{b}, \mathrm{c}}$, Choukri \\ Mekkaoui $^{\mathrm{b}, \mathrm{c}}$, Lawrence L Wald ${ }^{\mathrm{b}, \mathrm{c}, \mathrm{d}}$, Susie Y Huang ${ }^{\mathrm{b}, \mathrm{c}, \mathrm{d}}$, Anastasia Yendiki ${ }^{\mathrm{b}, \mathrm{c}}$, Boris Keil ${ }^{\mathrm{a}, \mathrm{e}}$ \\ ${ }^{a}$ Institute of Medical Physics and Radiation Protection (IMPS), TH-Mittelhessen University of Applied Sciences \\ (THM), Giessen, Germany \\ ${ }^{b}$ A.A. Martinos Center for Biomedical Imaging, Department of Radiology, Massachusetts General Hospital, \\ Boston, Massachusetts, USA. \\ ${ }^{c}$ Harvard Medical School, Boston, Massachusetts, USA. \\ ${ }^{d}$ Harvard-MIT Division of Health Sciences and Technology, Cambridge, Massachusetts, USA. \\ ${ }^{e}$ Center for Mind, Brain and Behavior (CMBB), Marburg, Germany.
}

\begin{abstract}
In vivo diffusion-weighted magnetic resonance imaging is limited in signal-to-noise-ratio (SNR) and acquisition time, which constrains spatial resolution to the macroscale regime. Ex vivo imaging, which allows for arbitrarily long scan times, is critical for exploring human brain structure in the mesoscale regime without loss of SNR. Standard head array coils designed for patients are suboptimal for imaging ex vivo whole brain specimens. The goal of this work was to design and construct a 48-channel ex vivo whole brain array coil for high-resolution and high $b$-value diffusionweighted imaging on a $3 \mathrm{~T}$ Connectome scanner. The coil was validated with bench measurements and characterized by imaging metrics on an agar brain phantom and an ex vivo human brain sample. The two-segment coil former was constructed for a close fit to a whole human brain, with small receive elements distributed over the entire brain. Imaging tests including SNR and G-factor maps were compared to a 64-channel head coil designed for in vivo use. There was a 2.9-fold increase in SNR in the peripheral cortex and a 1.3-fold gain in the center when compared to the 64 -ch head coil. The 48-channel ex vivo whole brain coil also decreases noise amplification in highly parallel imaging, allowing acceleration factors of approximately one unit higher for a given noise amplification level. The acquired diffusion-weighted images in a whole ex vivo brain specimen demonstrate the applicability of the developed coil for high-resolution and high $b$-value diffusion-weighted ex vivo brain MRI studies.
\end{abstract}

\footnotetext{
${ }^{\star}$ Funding: This work was supported by the National Institutes of Health [R01EB021265, 1U01EB026996, R01HL131635]; and the Federal Ministry of Education and Research Germany (BMBF) [IN2016-2-226].

${ }^{*}$ Corresponding Author: TH-Mittelhessen University of Applied Sciences (THM), 14 Wiesenstrasse, 35390 Giessen, Germany, alina.scholz@lse.thm.de
} 
bioRxiv preprint doi: https://doi.org/10.1101/2021.02.24.432713; this version posted February 25, 2021. The copyright holder for this preprint (which was not certified by peer review) is the author/funder, who has granted bioRxiv a license to display the preprint in perpetuity. It is made available under aCC-BY-NC-ND 4.0 International license.

Keywords: Magnetic Resonance Imaging, Diffusion-Weighted Imaging, RF coil, receive array coil, brain imaging, ex vivo brain 


\section{Abbrevations}

$48 \mathrm{ch}$ 48-channel

64ch 64-channel

BW bandwidth

5 CAD computer aided design

dMRI Diffusion MRI

DTI Diffusion Tensor Imaging

EPI echo planar imaging

EPROM Erasable Programmable Read-Only Memory

10 F flip angle

FA fractional anisotropy

FOV field of view

M matrix

PC polycarbonate

15 PCB printed circuit board

PD proton density

PLP periodate-lysine-paraformaldehyde

$\mathbf{R F}$ radio frequency

ROI region of interest

2o SMS simultaneous multislice

SNR signal-to-noise-ratio

TE echo time

TR repetition time

VNA vector network analyzer 


\section{Introduction}

Diffusion MRI (dMRI) is a powerful, non-invasive technique for imaging axonal orientations as well as characterizing white and gray matter microstructure [15]. The basic premise of dMRI in the human brain is that the diffusion of water molecules in white matter is anisotropic, and that its preferential direction is aligned with the orientation of the underlying fibers [2]. A series of images, each sensitized to diffusion in a different direction, are acquired and used to infer the most likely orientation of water displacement in every voxel [6].

There are several requirements that increase the acquisition time needed for whole-brain dMRI. High spatial resolution is desirable for resolving small brain structures. A large number diffusion directions must be sampled to improve the angular resolution, i.e., the smallest angle between crossing fiber bundles that can be resolved. Finally, advanced dMRI sampling schemes may require images to be acquired with multiple $b$-values. Satisfying all these requirements would lead to acquisition times that are prohibitive for in vivo imaging in the absence of any image acceleration. As a result, trade-offs must be made that restrict in vivo whole-brain dMRI to the macroscale regime [4, 7], with voxel sizes on the order of 1 to $3 \mathrm{~mm}$. Motion artifacts, which are exacerbated by long acquisitions, and distortions near tissue-air interfaces further degrade the effective resolution that is achievable in vivo.

Many of these issues can be circumvented in ex vivo dMRI, which allows for longer acquisition times, absence of motion and significantly reduced susceptibility artifacts with appropriate sample preparation [8]. Furthermore, ex vivo imaging enables the placement of coil elements closer to the actual brain tissue to maximize sensitivity. Thus, ex vivo imaging can achieve substantially higher spatial and angular resolution, permitting the anatomy and microstructure of complex fiber pathways to be imaged at the mesoscale, sub-millimeter regime, well beyond what is feasible in vivo. The impressive level of anatomical detail that can be resolved by ex vivo dMRI has already been demonstrated on a variety of human tissue samples [9 13]. Ex vivo dMRI, in combination with optical imaging, is an excellent tool for validating dMRI acquisition and analysis methods in human brain tissue [14, 15].

Higher spatial resolution comes at the cost of lower signal-to-noise-ratio (SNR). Several strategies for improving SNR in high-resolution ex vivo dMRI have been proposed and tested, mainly focusing on higher magnetic field strengths [16, 17], small-bore MRI scanners [18, 19] or high-performance gradient systems [20]. One of the main innovations introduced by the NIH Blueprint Human Connectome Project was the development of human scanners with ultra-high gradients, which allow high $b$-values to be achieved without loss of SNR [21]. Initial results have already shown the advantages of a $300 \mathrm{mT} \mathrm{m}^{-1}$ gradient system for imaging whole post-mortem human brains at $0.6 \mathrm{~mm}$ isotropic resolution [20], or smaller, non-human primate brain samples at $0.8 \mathrm{~mm}$ isotropic resolution [22. Those results were obtained with an in vivo head coil. Dedicated ex vivo brain 
coils are known to increase signal reception sensitivity, and a few studies have shown the benefits of multi-channel brain array coils for ex vivo tissue imaging applications [23 25].

The aim of this study was to push the limits of spatial and angular resolution in ex vivo dMRI by designing, constructing, and validating a 48-channel $(48 \mathrm{ch})$ receive array coil for ex vivo whole human brain examinations. The array coil was developed for high spatial resolution and high $b$ value dMRI acquisitions with long scan times (a few hours to a few days) on the $3 \mathrm{~T}$ Connectome scanner [20, 21]. This work presents high-sensitivity ex vivo diffusion MRI results obtained in a whole human brain specimen at mesoscale resolution ( $0.73 \mathrm{~mm}$ isotropic) using the $48 \mathrm{ch}$ receive coil on the $3 \mathrm{~T}$ Connectome scanner and expands on preliminary results that were published in conference proceedings [26].

\section{Material and Methods}

\subsection{Coil Design and Construction}

To closely cover a whole human brain, we designed an anatomically-shaped ex vivo brain coil former (Figure 1 and 2 based on a nonlinear brain atlas of the International Consortium for Brain Mapping (ICBM). The coil housing was modeled with 3D computer aided design (CAD) software (Rhino3D, Robert McNeel \& Associates, Seattle, WA, USA, version 6). It was designed to completely surround the brain with minimal space between the coil elements and imaging volume. The coil former was split into an upper and lower part, such that a whole brain can be placed inside the coil container. Both coil segments close with an overlapping rim structure. The coil container can accommodate whole brains with dimensions of $140 \mathrm{~mm}$ in the left-to-right direction, an anteriorto-posterior diameter of $182 \mathrm{~mm}$, and a superior-inferior distance of $110 \mathrm{~mm}$. The completed array coil is shown in Figure 3

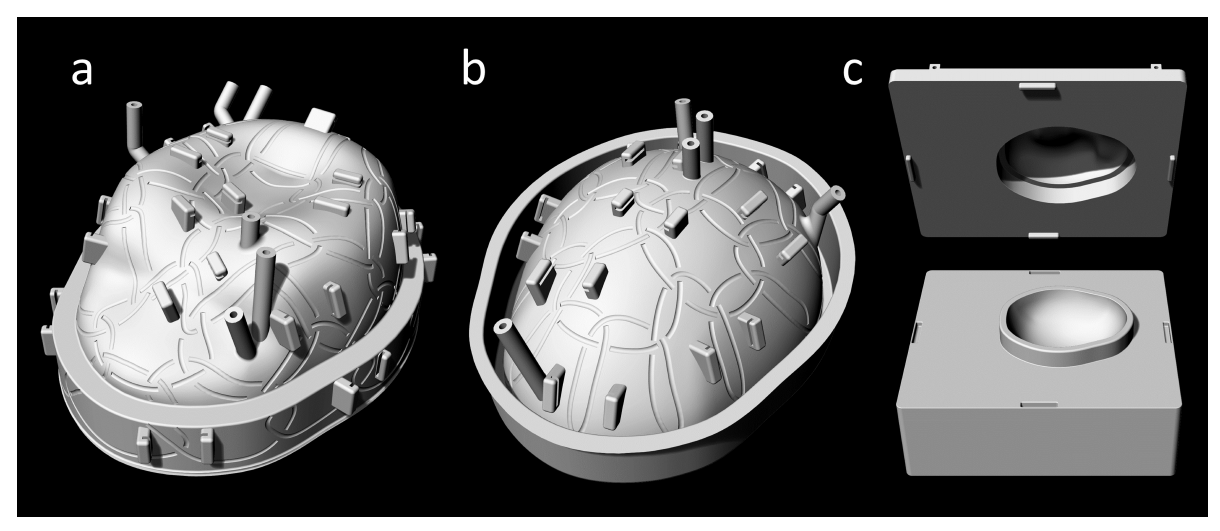

Figure 1: computer aided design model of the coilformer with graved loops and standoffs for preamplifier boards. (a) Top coilformer part (b) Bottom coilformer part (c) Inner side of both coilformer parts with overlapping frames to allow geometrical decoupling of the loops from top and bottom part. 


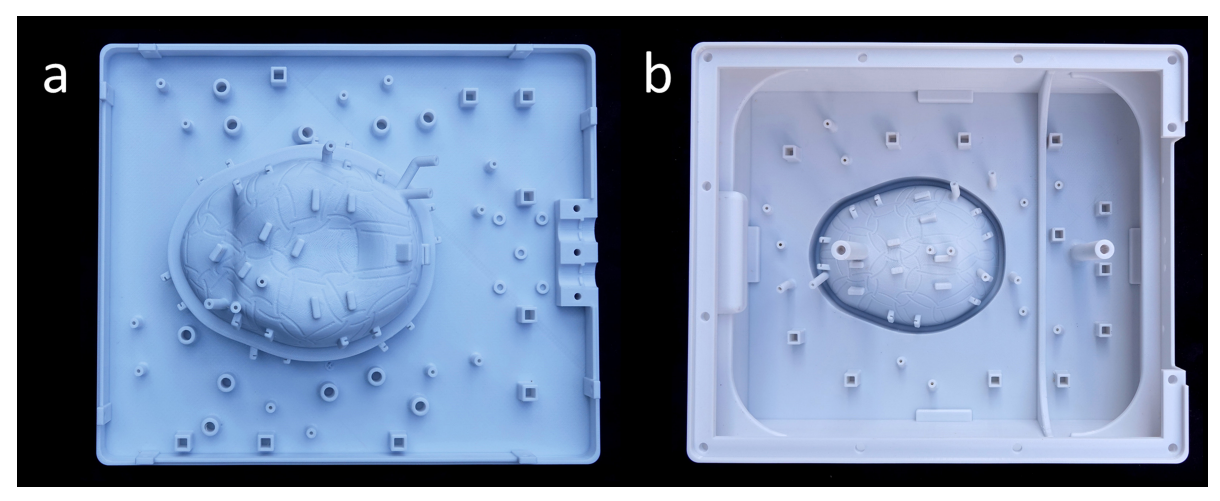

Figure 2: Polycarbonate printed coilformer with standoffs for preamplifier boards. (a) Top coilformer part. (b) Bottom coilformer part, in addition to two pillars to handle the weight of the brain and top part.

In the bottom coil segment, we incorporated the mechanics for a plugging slide mechanism (Figure $3 \mathrm{~b}$ and $3 \mathrm{~d}$ ), which directly plugs it into the scanner's patient bed. The top coil segment is connected to the scanner using two standard multi-channel coil plugs. The ex vivo coil container was designed to allow the brain to be placed at the isocenter of the scanner.

The positions of the 48 coil elements on the outer surface of the coil former were derived from a hexagonal/pentagonal tiling pattern [27], with 30 and 18 coil elements located on the top and bottom segments, respectively (Figure 44.The position and outline of all loop elements, which are decoupled geometrically from neighboring loops by critical overlap [28], were incorporated in the CAD model. The majority of the loops was circularly formed, whereas some loops were arbitrarily shaped to fit over the rim structure. The loop size and thus the critical overlap was determined empirically in previously tested bench measurements. The average diameter of the circular coil elements is $54 \mathrm{~mm}$ with an inductance of about $203 \mathrm{nH}$. The overlap of these loops is about 0.27 times the loop diameter. Standoffs for circuit boards and cable routing were implemented to provide stable mounting positions. The coil former including its cover were then 3D-printed in polycarbonate (PC) using a 3D printer (Fortus 350, Stratasys, Eden Prairie, USA).

\subsection{Coil Circuit}

The loop elements were constructed out of $1.3 \mathrm{~mm}$ thick tin-coated copper wire. Compared to flat circuit board copper traces, the wire loops reduce eddy current losses in a high-density array coil architecture 29]. Implemented small bridges in the conductor enable one loop to cross over another without touching [30].

Each coil circuit (Figure 5) consists of a loop with three symmetrically placed ceramic capacitors (Series 11, Voltronics, Danville, NJ), one variable plastic capacitor (GFX2700NM; Sprague Goodman, Westbury, New York, USA), a matching network to the preamplifier (Siemens AG, Healthineers, Erlangen, Germany), and an actively controllable detuning resonant circuit. A typically redundant 
bioRxiv preprint doi: https://doi.org/10.1101/2021.02.24.432713; this version posted February 25, 2021. The copyright holder for this preprint (which was not certified by peer review) is the author/funder, who has granted bioRxiv a license to display the preprint in perpetuity. It is made available under aCC-BY-NC-ND 4.0 International license.

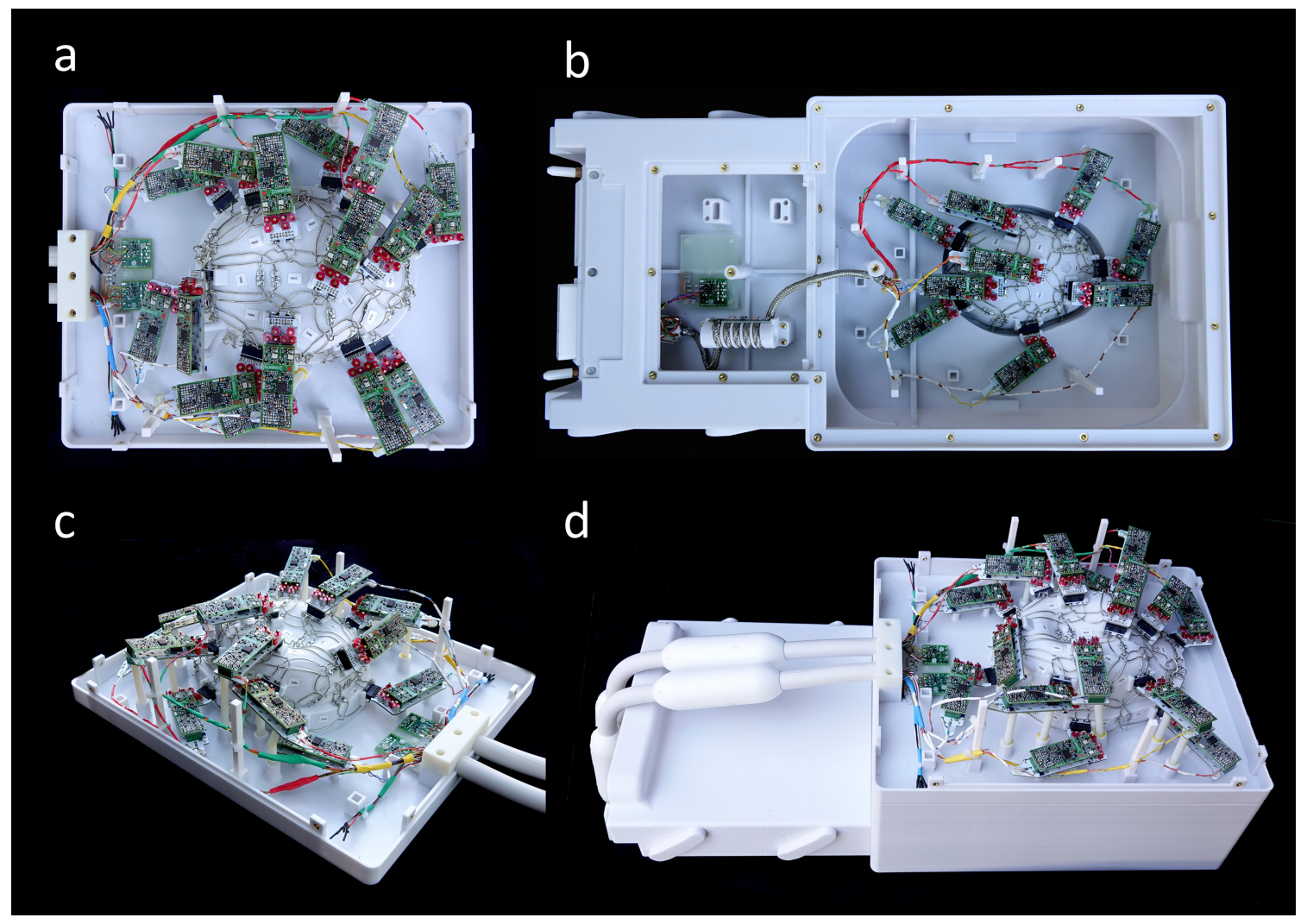

Figure 3: Completed coil with loops, preamplifier boards, preamplifiers, cable trap and EPROMs without coil covers (a) and (c) Top part with 30 coil elements (b) Bottom segment with 18 coil elements and a cable trap. (d) Assembled coil consisting of the top part and bottom part. The scanner connection cables from the top part involve two cable traps each and standard coil plugs. The bottom part uses a sliding connection mechanism.

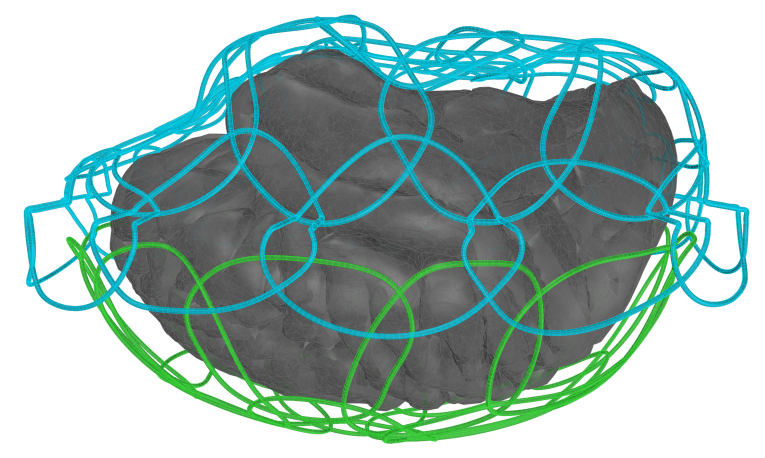

Figure 4: Placement of the 30 top loops (blue) and the 18 bottom loops (green) around the brain (gray) in the computer aided design program. 


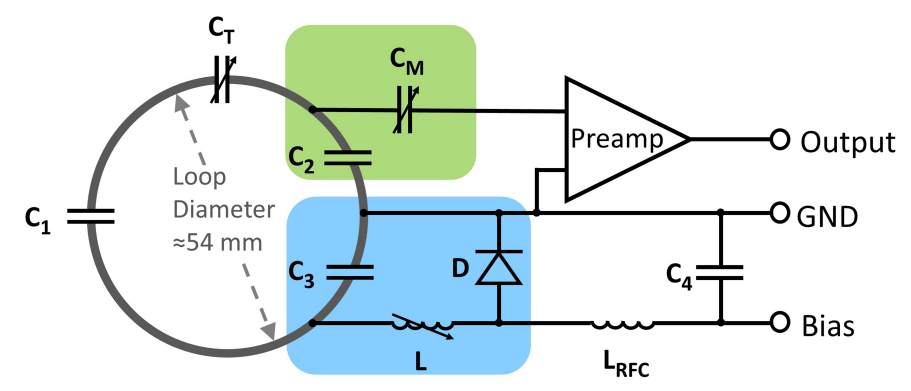

Figure 5: Circuit schematic for one coil element. Each loop consists of three fixed capacitors $\left(C_{1}-C_{3}\right)$ and one variable capacitor $\left(C_{T}\right) . C_{T}$ fine-tunes the resonant frequency of the coil to Larmor frequency corresponding at $3 \mathrm{~T}$. $C_{2}$ and $C_{3}$ create a capacitive voltage divider. $C_{3}$ is part of the active detuning circuit (blue) together with the variable inductor $L$ and the PIN diode $D . C_{2}$ and $C_{M}$ (green) provide both impedance matching of the loop and impedance transformation to establish preamplifier decoupling. Typical values for the components are: $C_{1}=33 \mathrm{pF}$ , $C_{2}=56 \mathrm{pF}, C_{3}=56 \mathrm{pF}, C_{T} \approx 18 \mathrm{pF}, C_{M} \approx 18 \mathrm{pF}, L \approx 24.5 \mathrm{nH}, L_{R F C}=2.7 \mu \mathrm{H}$.

passive detuning safety mechanism for in vivo examinations was omitted for this ex vivo coil.

The variable capacitor $C_{T}(3-33 \mathrm{pF})$ was used to fine-tune the loop resonance to the Larmor frequency at $3 \mathrm{~T}(123.25 \mathrm{MHz}) . C_{2}$ and $C_{3}$ create a capacitive voltage divider. The variable capacitor $C_{M}(3-33 \mathrm{pF}$, GFX2700NM; Sprague Goodman, Westbury, New York, USA) provides impedance matching of the loop output to a $50 \Omega$ noise matched condition needed by the preamplifier to operate at the lowest noise figure at $123.25 \mathrm{MHz}$ ) [31. To ensure accurate detuning of the loop elements, an active detuning circuit was implemented. It consists of one of the voltage dividing capacitors $C_{3}$ and a variable inductor $L$ (Coilcraft Inc., $25-32 \mathrm{nH}, 165-02 \mathrm{~A} 06 \mathrm{~L}$, Cary, IL, USA) in series to a PIN diode D (MA4P4002B-402; Macom, Lowell, MA, USA) 32]. During transmit, a DC current is applied to forward bias the PIN diode. This in turn activates the detuning resonant circuit at the Larmor frequency and generates a high impedance in the loop to suppress current flow. The RF-choke $L_{R F C}$ (Coilcraft Inc, $2.7 \mu \mathrm{H}, 1812 \mathrm{CS}-333 \mathrm{XJLC}$ Cary, IL, USA) and $C_{4}$ block the RF signal to prevent passing into bias source.

While nearest neighbors use geometrical decoupling, next-nearest neighbors and further coil elements are decoupled by the impedance transformation of the input of the preamplifiers [28]. The capacitors $C_{2}$ and $C_{M}$ and the preamplifier's input impedance form a resonant circuit, which enables a voltage-source measurement setup, where RF current flow is minimized. As a consequence, inductive coupling across elements is highly reduced and all coil elements receive independently, while maintaining a $50 \Omega$ output impedance.

Both the matching and detuning network of the coil element are placed on the preamplifier's daughter board, rather than soldering these components directly to the coil former. Therefore, the daughter board is a part of the coil element. The printed circuit board (PCB) daughter board is connected to the loop with an intermittent pin connector. This setup allows a fast construction process of dense array coils. 
According to the RF scanner architecture, pre-amplified signals from two loops elements are multiplexed onto one output coaxial cable. The bundled output cables are passed through cable traps to prevent RF common mode currents on the shield of the coaxial cable [33]. The cable traps comprise a wounded coaxial cable bundle, which form an inductance $(\approx 109 \mathrm{nH})$, and a parallel ceramic high power capacitor (15.2 pF, Series 25, Voltronics, Danville, NJ), which resonates at Larmor frequency. Two traps are incorporated into the cables of the upper array coil segment and one cable trap is located directly in the bottom coil housing part.

\subsection{Coil Bench Measurements}

For bench measurements during the construction process, a custom-made coil plug simulator was used. It provides voltage for the preamplifiers $(3 \mathrm{~V})$ and the opportunity to apply a DC current $(100 \mathrm{~mA})$ to bias manually each PIN diode forward, which allows for active detuning of single coil elements. To gather information about bench level metrics, e.g. transmission and reflection measurements, a vector network analyzer (VNA) (ENA series, Agilent Technologies, Santa Clara, CA) and custom-built RF tools such as single / double probes and sniffer probes were used. These measurements included tuning to Larmor frequency, active detuning, preamplifier decoupling and geometrical nearest neighbor decoupling of each coil element.

The loops were tuned under a $S_{21}$ control with a $50 \Omega$ dummy load plugged into the preamplifier socket, while all other elements of the array were detuned. Active detuning was performed by using $S_{21}$ measurement with the double-probe for each loop, while all other coil elements were detuned and the relevant loop under test was switched between the tuned and detuned state. The difference of both states at the Larmor frequency indicates the magnitude of active detuning. A similar $S_{21}$ double-probe measurement was carried out to determine the effectiveness of the implemented preamplifier decoupling, first by plugging the preamplifier into the socket on the PCB and second by terminating the socket with a load impedance of $50 \Omega$. Again, all but the loop element to be tested were detuned.

Coupling of nearest neighbor elements was measured with direct $S_{21}$ VNA measurement by using coaxial cables, which were directly plugged into the preamplifier sockets. During this measurement, all other coil elements were detuned. This measurement configuration was also used to verify $50 \Omega$ coil impedance matching using $S_{11}$ and $S_{22}$ measurements. [30, 31]

Furthermore, unloaded-to-loaded coil quality factor ratio $\left(Q_{U} / Q_{L}\right)$ of one representative coil element was measured within the populated but detuned array assembly, using the $S_{21}$ double-probe method [34]. As a load, a fixed tissue brain sample in periodate-lysine-paraformaldehyde (PLP) solution was used. 


\subsection{MRI data acquisition and analysis}

Imaging metrics were acquired on a clinical 3T MRI scanner (MAGNETOM, Skyra, Tim 4G, Dual Density Signal Transfer, Siemens AG, Healthineers, Erlangen, Germany), equipped with a customized gradient coil (AS302 CONNECTOM 1.0 gradient) 11 with a maximum gradient strength of $300 \mathrm{mT} \mathrm{m}^{-1}$ and a maximum slew rate of $200 \mathrm{~T} \mathrm{~m}^{-1} \mathrm{~s}^{-1}$.

For evaluating the developed ex vivo whole brain array coil, we constructed a human-brain-shaped phantom using a 3D printer (Objet30 Pro, Stratasys, Eden Prairie, USA). The phantom was filled with a mixture of $830 \mathrm{ml}$ distilled $\mathrm{H}_{2} \mathrm{O}, 29 \mathrm{~g} \mathrm{NaCl}, 12.5 \mathrm{~g}$ of agar powder (Sigma-Aldrich Corp., St. Louis, MO) and $936 \mathrm{~g}$ sugar [35]. The electrical characteristics of the brain phantom were determined with a VNA equipped with a dielectric probe kit (85070E kit, Agilent Technologies, Santa Clara, CA) and were representative of the averaged human brain $\left(\epsilon_{r}=66.34\right.$ and $\left.\sigma=0.49 \mathrm{~S} \mathrm{~m}^{-1}\right)$.

For determining SNR and G-factor, the phantom was scanned with a proton density (PD)-weighted FLASH sequence (repetition time $(\mathrm{TR})=200 \mathrm{~ms}$, echo time $(\mathrm{TE})=4.8 \mathrm{~ms}$, flip angle $(\mathrm{F})=15^{\circ}$, matrix (M): $192 \times 192(\mathrm{SNR})$ and $64 \times 64$ (G-factor and SNR in parallel imaging), field of view (FOV): $256 \times 256 \mathrm{~mm}^{2}$, slice thickness: $8 \mathrm{~mm}$, bandwidth $(\mathrm{BW}): 200 \mathrm{~Hz} /$ pixel). Information about noise correlation was obtained with the same sequence but without RF excitation.

Pixel-wise SNR maps were calculated using the noise-covariance-weighted, root sum-of-squares image reconstruction method from Kellman et al. [36. To evaluate the array coil's encoding capability for parallel imaging, SENSE G-factor maps were computed using the acquired noise correlation matrix and complex sensitivities of the coil elements [37]. The FOV of the G-maps was tightly enclosed to the phantom, in order to enhance the aliasing pattern inside the imaging object.

A valuable metric is the remaining image SNR after the parallel imaging acceleration has been performed. We calculated the remaining SNR by dividing the SNR globally by the square root of the reduction factor $R$ and further locally with the noise amplification given by the G-factor.

For further characterization of the coil performance, we examined the encoding power for simultaneous multislice (SMS) acquisitions with blipped-controlled aliasing in parallel imaging [38 40]. To assess the encoding capability of combined SMS and in-plane acceleration, a reduction factor of $R=2$ and a slice acceleration factor from $M B=4$ up to $M B=8$ with a $1 / 3 \mathrm{FOV}$ shift were evaluated. Noise correlation and SNR and G-factor maps of the $48 \mathrm{ch}$ ex vivo brain coil were compared to a customized 64-channel (64ch) whole head receive array coil [41] with identical acquisition parameters.

In addition, time course stability of each coil element was measured with a single-shot gradient echo echo planar imaging (EPI) sequence (time points: $500, \mathrm{TR}=1000 \mathrm{~ms}, \mathrm{TE}=30 \mathrm{~ms}, \mathrm{~F}=90^{\circ}$, M: $64 \times 64$, FOV: $200 \times 200 \mathrm{~mm}^{2}$, slices: 16 slices of $15 \mathrm{~mm}$, BW: $2298 \mathrm{~Hz} \mathrm{px}^{-1}$ ) with the brain phantom. The average intensity of a 15-pixel square region of interest (ROI) in the brain center

\footnotetext{
${ }^{1}$ under development and not commercially available in the U.S. and its future availability cannot be assured.
} 
was de-trended with linear and quadratic temporal trends and plotted. The stability was calculated as the variation of signal intensity from peak-to-peak as a percentage from the average signal intensity 42 .

Finally high-resolution ( $0.73 \mathrm{~mm}$ isotropic) diffusion imaging was performed on a whole, ex vivo human brain. The brain had been excised from a male who had died of non-neurological causes, and had been placed in fixative ( $10 \%$ formaldehyde) for 90 days before being transferred to paraformaldehyde-lysine-periodate solution for long-term storage. Diffusion-weighted images were acquired with a 3D diffusion-weighted spin echo echo planar imaging multi-shot sequence ( $\mathrm{TR}=500 \mathrm{~ms}$, TE $=65 \mathrm{~ms}$, echo spacing: $1.22 \mathrm{~ms}$, M: $160 \times 268 \times 208$, FOV: $117 \times 196 \mathrm{~mm}^{2}$, BW: $1244 \mathrm{~Hz} \mathrm{px}^{-1}, 16$ shots, EPI factor $=10$, no partial Fourier, maximum gradient strength of $91 \mathrm{mT} \mathrm{m}^{-1}$ ). One image with $b=0$ and 16 diffusion-weighted volumes with $b=4000 \mathrm{~s} \mathrm{~mm}^{-2}$ and non-colinear diffusion encoding directions were acquired. Diffusion-weighted volumes were corrected for eddy current distortions with the $e d d y$ tool from FSL [43]. The diffusion tensor model was fit by linear least-squares fitting of the logarithm of the dMRI signal with the dtifit tool in FSL. The phase-encoding direction was anterior-posterior when considering the conventional sagittal plane. Since the brain in the constructed coil was rotated compared to the usual orientation of a patient, the anatomical axis of the phase-encoding direction was inferior-superior.

\section{Results}

\subsection{Coil Bench Measurements}

The $Q_{U} / Q_{L}$-ratio of a $54 \mathrm{~mm}$ loop element was measured to be $233 / 46=5.1$ with six surrounding but non-resonant neighboring loops. Thus, the array's loop elements operate in the sample noise dominated regime. The geometrical decoupling of nearest neighbors was $S_{21}$ measured with an average value of $-16 \mathrm{~dB}$ and ranged from $-14 \mathrm{~dB}$ to $-18 \mathrm{~dB}$. Non-adjacent and thus non-overlapping coil elements, which are primarily decoupled via preamplifier decoupling, obtained an average decoupling value of $-18 \mathrm{~dB}$ with a range from $-17 \mathrm{~dB}$ to $-19 \mathrm{~dB}$. The isolation between tuned and detuned states caused by the active detuning circuit reached an average value of $42 \mathrm{~dB}$.

\subsection{Image Performance}

Figure 6 shows the noise correlation matrix of the $48 \mathrm{ch}$ ex vivo coil and that of the $64 \mathrm{ch}$ in vivo coil. The ex vivo array has a range of noise correlations from $0.02 \%$ to $35.8 \%$ with an average value of $7.5 \%$, while the in vivo array has noise correlations from $0.12 \%$ to $53.8 \%$ with an average value of $7.1 \%$ for the off-diagonal elements.

Figure 7 compares the SNR maps from the newly developed 48ch ex vivo brain coil to that of the existing, custom $64 \mathrm{ch}$ whole head coil, in different planes of the agar phantom. For both coils, the measured SNR is highest in the outer periphery and decreases towards the phantom center. The 
bioRxiv preprint doi: https://doi.org/10.1101/2021.02.24.432713; this version posted February 25, 2021. The copyright holder for this preprint (which was not certified by peer review) is the author/funder, who has granted bioRxiv a license to display the preprint in perpetuity. It is made available under aCC-BY-NC-ND 4.0 International license.

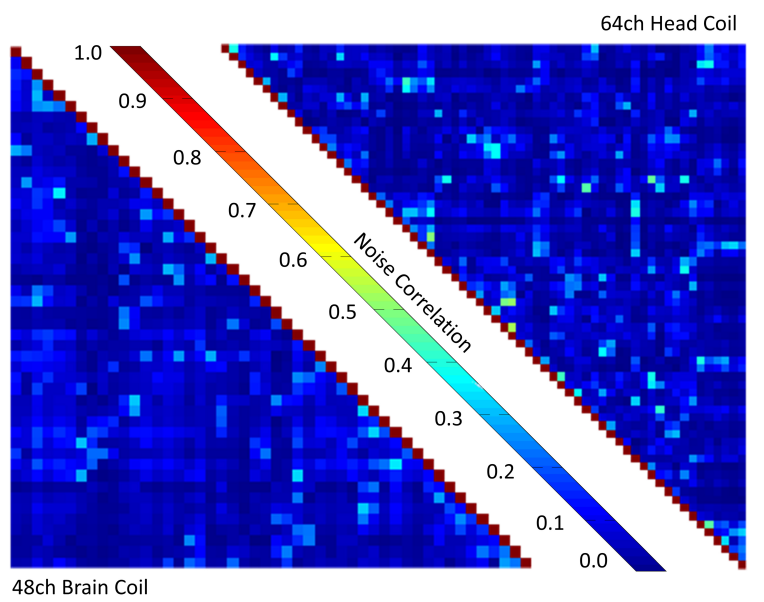

Figure 6: Noise correlation matrix of the $48 \mathrm{ch}$ ex vivo brain coil and the $64 \mathrm{ch}$ in vivo head coil with the scale normalized to 1.
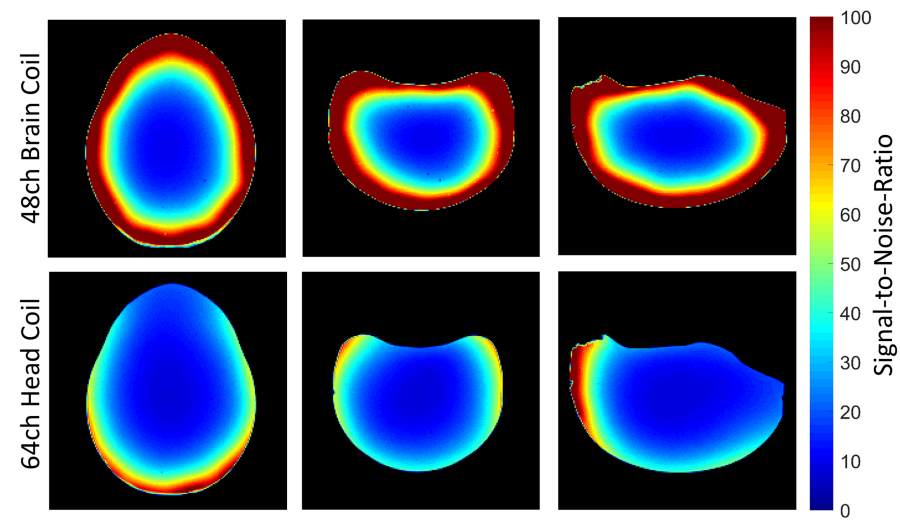

Figure 7: Comparison of the signal-to-noise-ratio, normalized to 100, of a transverse (left), coronal (middle) and sagittal (right) slice of the brain phantom with the $48 \mathrm{ch}$ ex vivo brain coil (top row) and the 64ch head coil (bottom row). The $48 \mathrm{ch}$ ex vivo brain coil shows a 1.3 -fold SNR gain in the center and a 2.9-fold SNR improvement in the peripheral regions when compared to the $64 \mathrm{ch}$ head coil. 


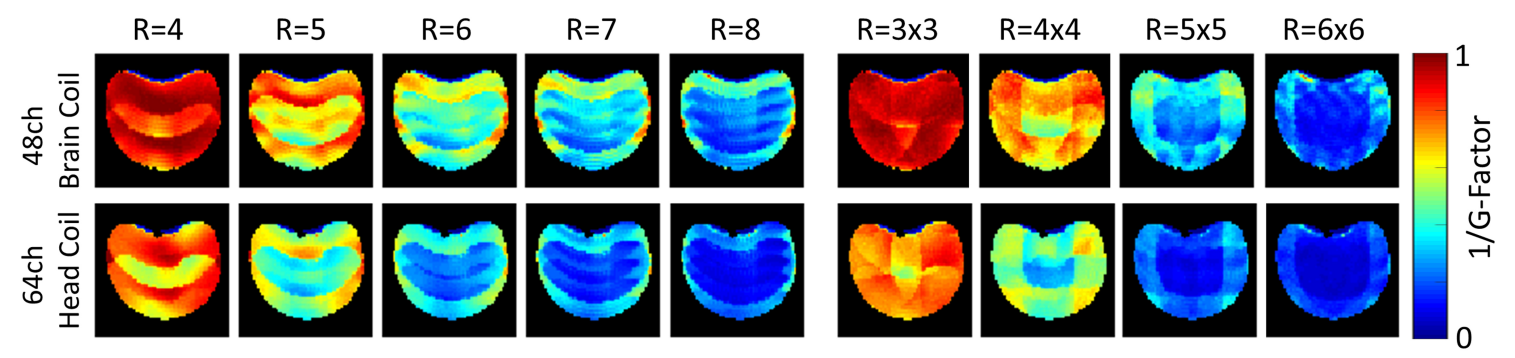

Figure 8: Comparison of inverse phantom G-factor maps between the $48 \mathrm{ch}$ ex vivo brain coil (top row) and the $64 \mathrm{ch}$ head coil (bottom row) for different acceleration factors $(R)$ obtained from representative coronal slice. The G-Factors from the $48 \mathrm{ch}$ ex vivo brain coil show overall lower noise amplification, when compared to the $64 \mathrm{ch}$ head coil.

newly constructed 48ch array coil outperforms the larger $64 \mathrm{ch}$ in vivo head coil by a factor of 2.5 , when the average SNR over the whole brain phantom volume is considered. In the periphery and in the center of the phantom, a 2.9-fold and 1.3-fold SNR gain was measured, respectively.

Figure 8 shows the SENSE inverse G-factor maps in a representative coronal plane of the brain phantom for both one-dimensional and two-dimensional acceleration obtained from the $48 \mathrm{ch}$ ex vivo brain coil and the $64 \mathrm{ch}$ in vivo head coil. The newly constructed $48 \mathrm{ch}$ coil provides significant improvement compared to the $64 \mathrm{ch}$ head coil for both in-plane acceleration types. Both coils show minimal noise amplifications for acceleration factors of $R=2, R=3$ and $R=2 \times 2$. However, for higher accelerations $(R>3)$ the $48 \mathrm{ch}$ ex vivo coil provides favorable encoding capabilities when compared to the $64 \mathrm{ch}$ in vivo head coil. At $R=4$, the $48 \mathrm{ch}$ coil shows on average a $16 \%$ lower G-factor than the $64 \mathrm{ch}$ head coil. When comparing the peak G-factors between both, the $48 \mathrm{ch}$ coil shows a $21 \%$ improvement. The enhanced encoding power of the $48 \mathrm{ch}$ coil becomes even more apparent when very high acceleration factors are compared. The improved average and peak Gfactor for $R=7$ was measured to be $35 \%$ and $41 \%$ lower. At $R=5 \times 5$ the noise amplifications could be reduced on average by $43 \%$, while the peak G-factor decreased by $53 \%$.

A more meaningful figure of merit is the SNR obtained from the accelerated image, where both the under-sampled $k$-space trajectory and the local noise amplification were taken into account. Figure 9 illustrates the accelerated SNR for both coils using box plots and its corresponding average values. Since the constructed $48 \mathrm{ch}$ coil provides both a higher baseline SNR and lower G-factors, it highly outperforms the $64 \mathrm{ch}$ head coil across all acceleration scenarios. The average SNR from the 64ch coil only reaches the lower 25 th percentile of the $48 \mathrm{ch}$ ex vivo coil. Further, it should be noted that the relative gain in average SNR increases with higher acceleration factors (e.g., factor 2.4 for $R=2$ and 3.9 for $R=7$ ) for both one-dimensional and two-dimensional acceleration.

Figure 10 compares the inverse G-factor maps for the SMS image reconstruction technique from a coronal slice of the brain phantom. Compared to the $64 \mathrm{ch}$ head coil, the constructed $48 \mathrm{ch}$ coil indicates overall substantially lower noise amplification for the SMS examination, as well as for 

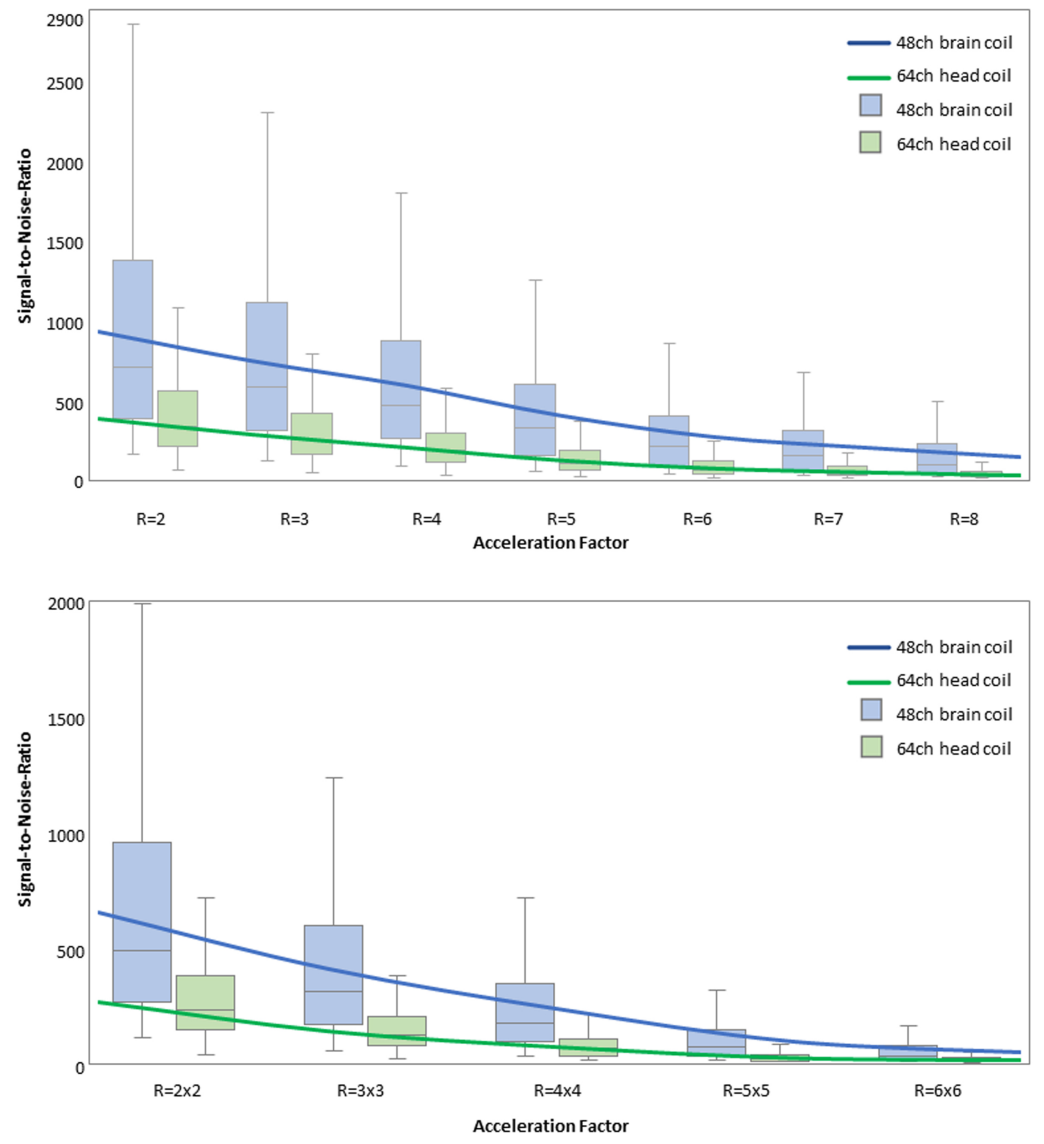

Figure 9: Parallel imaging accelerated signal-to-noise-ratio as a function of acceleration factor $(R)$ from the $48 \mathrm{ch}$ brain coil and the $64 \mathrm{ch}$ head coil for one-dimensional (top) and two-dimensional (bottom) accelerations. The continuous lines indicate the average SNR, box plots represent median (horizontal line), lower/upper quartiles and minimummaximum range (whiskers) without outliners. The constructed $48 \mathrm{ch}$ coil shows higher accelerated SNR in the entire range of acceleration factors. 
combined SMS and in-plane acceleration. At a multiband factor of $M B=4$, the $48 \mathrm{ch}$ coil generates negligible noise amplifications $\left(g_{\text {mean }}=1.0002\right.$ and $\left.g_{\max }=1.0569\right)$, while the $64 \mathrm{ch}$ head coil shows substantial noise gains of $g_{\text {mean }}=1.1218$ and $g_{\max }=1.6345$. Furthermore, the dedicated $48 \mathrm{ch}$ ex vivo brain coil achieves similar to slightly better encoding capabilities at $M B=8$ as the $64 \mathrm{ch}$ head coil at $M B=4\left(g_{\text {mean48ch }}=1.0047\right.$ vs. $g_{\text {mean64ch }}=1.1317$ and $g_{\text {max } 48 \mathrm{ch}}=1.2913$ vs. $\left.g_{\max 64 \mathrm{ch}}=1.6345\right)$. Therefore, the $48 \mathrm{ch}$ coil allows the application of a slice acceleration factor of $265 \quad M B=8$ with negligible noise gain.

To assess the accelerated SNR during SMS acquisitions, Fourier averaging needs to be taken into account: In the case of the $M B=8$ acceleration, eight times more ${ }^{1} \mathrm{H}$ spins are simultaneously excited compared with a single-slice acquisition. Thus, for a multiband factor $M B$, the SNR efficiency can be improved up to a factor of $\sqrt{ } M B$. This translates to an SNR increase by a factor 270 of up to $\sqrt{8} / g_{\max -48}=2.2$, when compared to a commonly used consecutive single-slice acquisition schemes. The $M B=8$ achievable SNR obtained from the $64 \mathrm{ch}$ is only increased by a factor of up to $\sqrt{8} / g_{\max -64}=1.2$. In direct comparison, when the baseline SNR, Fourier averaging, and G-factors are taken into account, the $48 \mathrm{ch}$ coil achieves up to a 4.5 -fold SNR improvement at $M B=8 \mathrm{com}$ pared to the $64 \mathrm{ch}$ head coil. Time course stability tests show a peak-to-peak variation of $0.4 \%$ over

275500 time-points EPI sequence measured in a ROI comprising $15 \times 15$ pixels.

As an initial demonstration of the high sensitivity images that can be acquired in ex vivo whole brain specimens using the $48 \mathrm{ch}$ coil, maps obtained from a $0.73 \mathrm{~mm}$ isotropic resolution dMRI scan of a whole fixed brain are shown in Figure 11. The five columns show: the $b=0$ image, a diffusion-weighted image (left-right diffusion-encoding direction), the mean diffusivity map, the 280 fractional anisotropy (FA) map, and the FA map color encoded by the principal eigenvector (V1) of the diffusion tensor. The figure includes a coronal view (a), with a magnified area showing fine anatomical detail in the striatum (b), and an axial view (c), with a magnified area showing radial fibers in the primary motor cortex (d). These maps illustrate the capability of our ex vivo coil to map detailed circuitry both in deep brain and near the cortical surface and demonstrate the

285 feasibility of obtaining data at high spatial resolution using the high gradient strengths available on the $3 \mathrm{~T}$ Connectome scanner.

\section{Discussion}

We designed, constructed, and evaluated a 48ch ex vivo brain array receive coil for high-resolution and high $b$-value dMRI of a whole ex vivo human brain on the $3 \mathrm{~T}$ Connectome scanner [20, 21. The coil was characterized by both bench tests and image metrics. Bench tests included element measurements of the coil quality factor $Q$, active detuning, geometrical decoupling, and preamplifier decoupling. MRI evaluations included measurements of the noise correlation, pixel-wise SNR, and G-factor, as well as time course stability using a brain shaped agar phantom. We demonstrated 
bioRxiv preprint doi: https://doi.org/10.1101/2021.02.24.432713; this version posted February 25, 2021. The copyright holder for this preprint (which was not certified by peer review) is the author/funder, who has granted bioRxiv a license to display the preprint in perpetuity. It is made available under aCC-BY-NC-ND 4.0 International license.
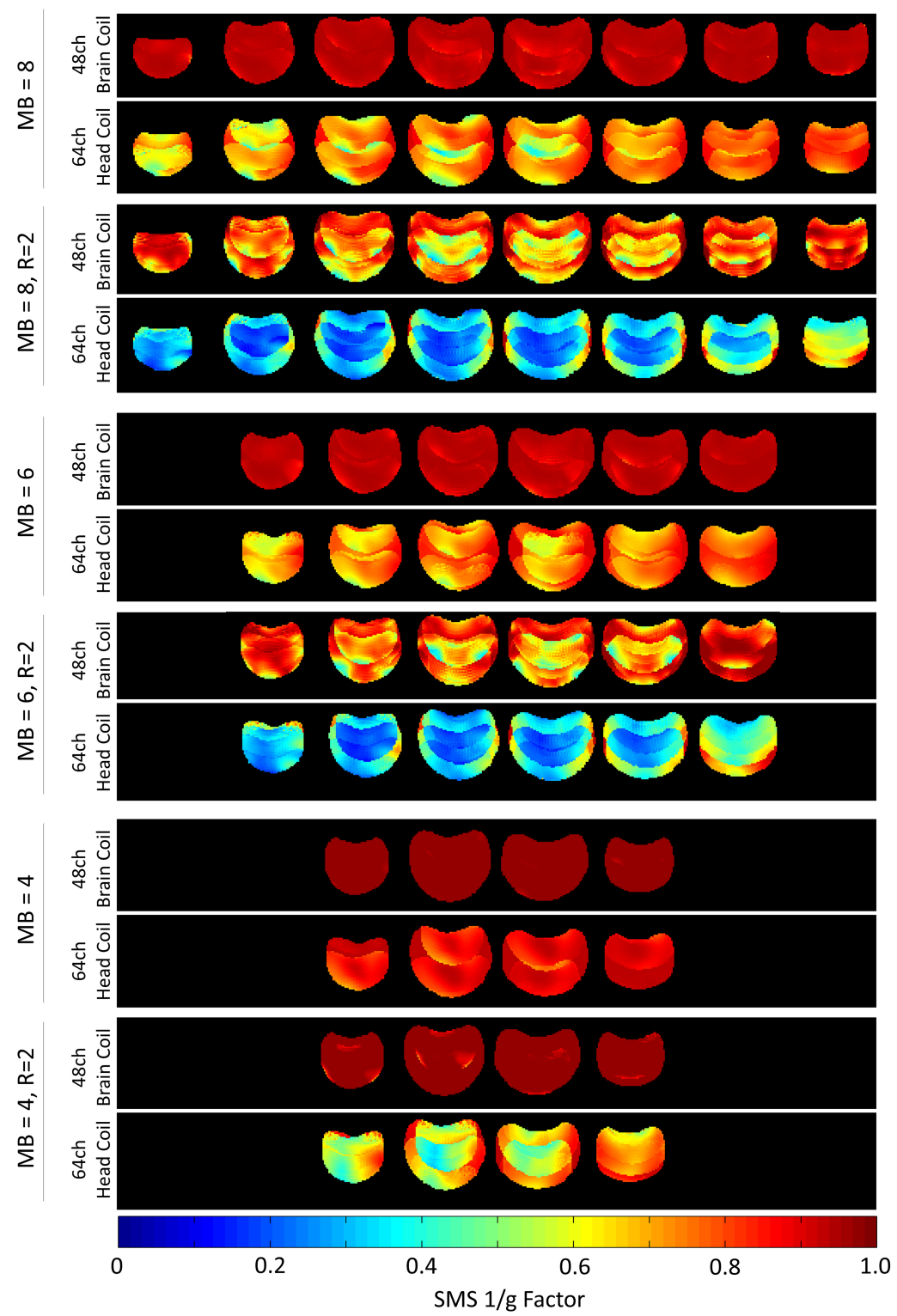

Figure 10: Comparison of inverse G-factor maps of the brain phantom for accelerated imaging with simultaneous multislice technique. The $48 \mathrm{ch}$ brain coil shows overall considerable lower noise amplification in comparison the the $64 \mathrm{ch}$ head coil. 
bioRxiv preprint doi: https://doi.org/10.1101/2021.02.24.432713; this version posted February 25, 2021. The copyright holder for this preprint (which was not certified by peer review) is the author/funder, who has granted bioRxiv a license to display the preprint in perpetuity. It is made available under aCC-BY-NC-ND 4.0 International license.

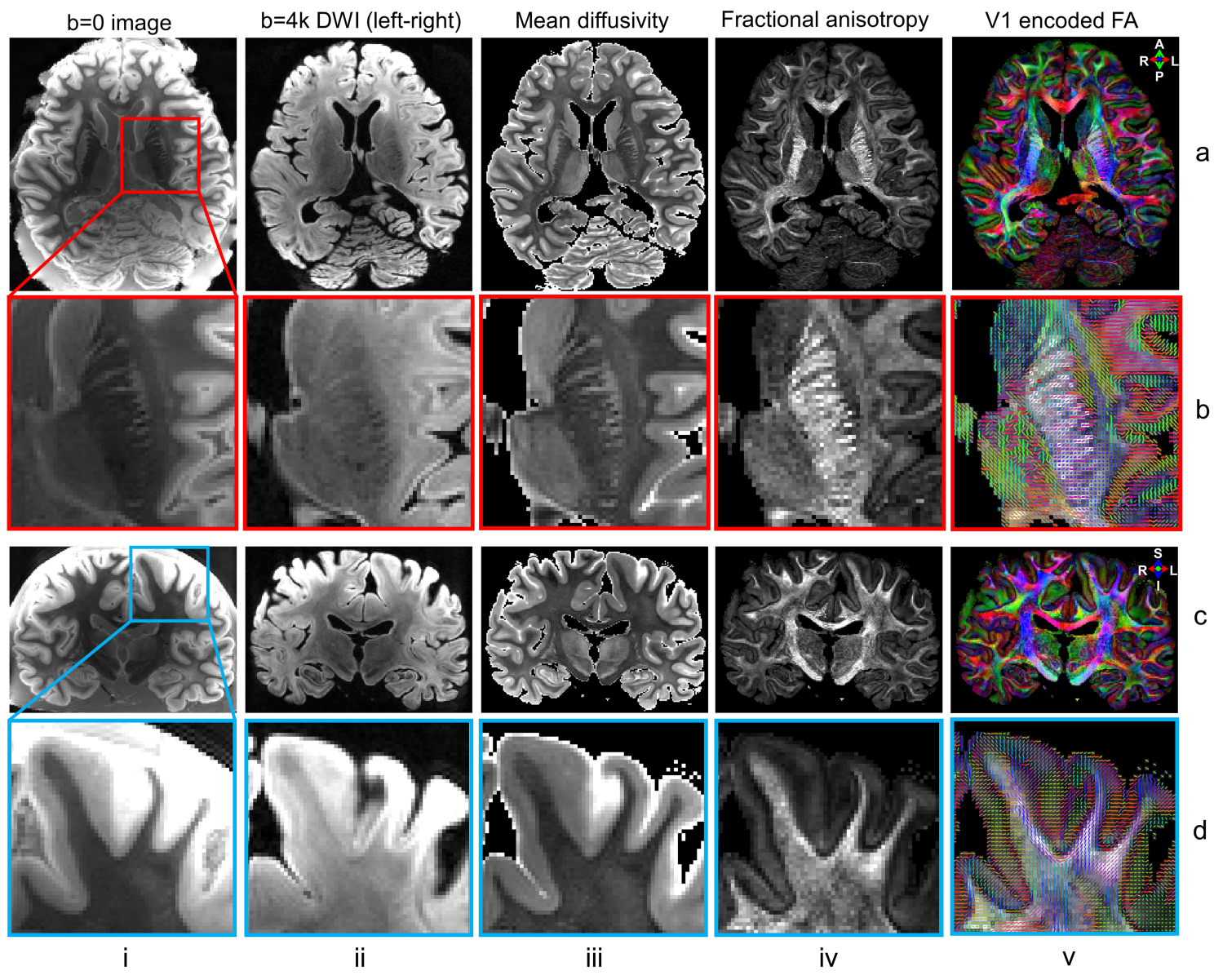

Figure 11: High-resolution Diffusion Tensor Imaging (DTI) results at $0.73 \mathrm{~mm}$ isotropic resolution with $b=0$ images (column i), diffusion-weighted images (DWI) acquired at $b=4000 \mathrm{~s} \mathrm{~mm}^{-2}$ along left-right diffusion-encoding direction (column ii), mean diffusivity maps (column iii), fractional anisotropy (FA) maps (column iv) and FA maps color encoded by the primary eigenvectors (V1) from DTI (column v). Two regions of interest in the deep white matter (red box) and sub-cortical white matter (blue box) are displayed in enlarged views (rows b and d) showing fine-scale structures in the internal capsule and cerebral cortex. 
the coil's performance in achieving high SNR with the acquisition of $0.73 \mathrm{~mm}$ isotropic resolution diffusion-weighted MR images of a whole ex vivo brain.

In many applications, large channel count arrays with relatively small loop sizes such as the $54 \mathrm{~mm}$ loops used here are necessary to increase both reception sensitivity and encoding power. However, very small loop elements quickly lose their sample noise dominance. Under these circumstances, small elements do not translate to higher SNR acquisitions anymore. For in vivo imaging at 3T, this critical size is reached at about $60 \mathrm{~mm}$ diameter [44. In ex vivo brain imaging, however, loop sizes can be made substantially smaller than for in vivo imaging. This is attributed to the brain fixation medium, which has a higher conductivity compared to in vivo tissue and thus provides a higher fraction of sample noise. While the noise increases in the ex vivo sample, the electronic noise can be decreased by omitting in vivo human safety features in the coil element circuity, such as passive detuning and RF-fuses. This condition results in an enhanced $Q_{U} / Q_{L}$-ratio when using small receiver elements. Therefore, the implemented loop size of $\approx 54 \mathrm{~mm}$ provides a relatively high $Q_{U} / Q_{L}$-ratio of 5.1, outperforming most coils optimized for in vivo applications with loop diameters ranging from $50 \mathrm{~mm}$ to $65 \mathrm{~mm}$ from our previous studies [41, 44, 45]. As a consequence, the minimum loop diameter at which sample noise dominance is maintained decreases for imaging fixed tissue brain samples in PLP solution, allowing us to contemplate very high-density arrays for ex vivo sample examinations.

Despite RF electrical optimizations, the mechanical coil former is an important and critical design aspect for ex vivo imaging. To improve SNR, the loops were populated very close to the sample, maximizing signal reception. Thus, the completely brain-enclosing coil former with uniformly distributed loop elements guarantees nearly omni-directional signal reception from the sample (decreased sensitivity was observed with some loops aligned to be almost parallel to the magnetic field $B_{0}$ ). However, an entirely surrounding coil array requires a split housing mechanism, which disturbs the loop layout and makes it difficult to maintain geometric decoupling at the split housing edge. Therefore, an overlapping edge structure was implemented, enabling adjacent loop elements to be geometrically decoupled across the two housing segments, while the overall array coil structure remains self-contained.

In array coil design, the central ultimate SNR is already approached with only 12 surrounding coil elements at $3 \mathrm{~T}$ [46. Implementing higher loop element counts only yields SNR improvements at the periphery for a given geometry. Nevertheless, relative central SNR gains are achievable with tightly fitting array coils. Due to the lack of dedicated ex vivo receiver arrays, in vivo head coils are commonly used in many ex vivo brain studies [3, 47-49]. However, these coils are not well suited in terms of sample fitting and SNR performance. Optimizing both the mechanical features for close fitting of samples and the RF circuitry can thus result in significant SNR gains in the brain. This implementation provides a $30 \%$ SNR increase of the $48 \mathrm{ch}$ coil at the phantom center when compared to the larger $64 \mathrm{ch}$ head coil. In addition, in the peripheral regions of the brain 
phantom, the tight-fitting form factor also provides favorable SNR gains, as evidenced by an almost 3 -fold SNR improvement over the 64ch coil. The high SNR can be exploited to reduce the voxel size, enabling high spatial resolution MR imaging of a whole ex vivo brain.

The average noise correlation of $9 \%$ indicates a well decoupled array and highly independent operating receiver loops. Adjacent loops show much higher coupling values up to $36 \%$, which can be attributed to insufficient overlap, resulting in a remaining mutual inductance and shared resistance especially in the sample voxels beneath the overlapping loop regions.

The constructed $48 \mathrm{ch}$ ex vivo brain coil shows remarkably better encoding performance when compared to the $64 \mathrm{ch}$ head coil. The encoding power of the $48 \mathrm{ch}$ coil enables approximately one additional acceleration unit, for both one-dimensional and two-dimensional accelerations, with the same noise amplification as the 64ch head coil. Improvements in G-factors are usually achieved by implementing higher channel counts on a given geometry. However, when comparing array coil formers of different sizes, similar improvements in G-factors can be achieved by (1) reducing the diameter of the coil elements at constant or even lower channel counts, and (2) positioning the coil elements in close proximity to the sample. The tight-fitting, smaller loop elements of the constructed 48ch coil provide an overall stronger spatial modulation in the signal sensitivity's magnitude and phase. Consequently, this coil arrangement allows favorable encoding capabilities for unaliasing folded images (SENSE method) or synthesizing spatial harmonics (GRAPPA or SMASH methods). Additionally, the entirely enclosed ex vivo coil former of the $48 \mathrm{ch}$ coil leads to better spatial coverage for the aliased pixels when compared to a head array coil, which obviously has limited coverage along the inferior aspect and in the area covering the face.

Reducing scan time using parallel imaging techniques is not strictly essential when constraints on acquisition time are lifted for ex vivo examinations. On the other hand, 2D acquisitions are still often used despite their SNR inefficiency per unit time [11, 22]. For example, mapping tissue microstructural features such as axon diameter throughout the whole human brain involves measurements at multiple $b$-values [50, and protocol optimization may be facilitated by 2D scans acquired at resolutions on the order of 0.8 to $1 \mathrm{~mm}$ isotropic. For such 2D acquisitions, slice acceleration enables the excitation and measurement of multiple slices [39, 40, 51]. Unlike conventional parallel imaging, which requires under-sampled data acquisition, these techniques provide acceleration by exciting the spins in multiple slices at the same time using multi-band radiofrequency pulses. These newer multi-band MR acquisitions have the SNR advantages of 3D sampling based on Fourier averaging [38, 39]. Therefore, SNR efficiency can be improved by up to a factor of $\sqrt{ } M B$. In practice, however, the SNR gain is reduced locally by the SMS G-factor of the coil and by changes in the sequence parameters, e.g., reduction of TR. The SNR recovery achieved by the SMS method is highly advantageous for dMRI, which normally suffers from low signal strength. Therefore, it is advantageous for ex vivo array coils to provide a high encoding capability for SMS in order to accommodate modern acquisition techniques. Commonly used in vivo head coils do not optimally 
fulfill this requirement for SMS ex vivo scans, as they lack enough elements in the $z$-direction. The radially surrounding, $z$-directional, stacked elements of the constructed coil provide favorable spatial coverage for SMS image encoding, allowing the separation of multiple collapsed slices. In the case of an $M B=8$ acceleration scheme, the combination of the enhanced SMS encoding power and the increased baseline SNR of the 48ch coil, provides an up to 4.5-fold SNR improvement when compared to the $64 \mathrm{ch}$ head coil.

Previous work comparing ex vivo dMRI to optical imaging suggests that high spatial resolution (1 mm or higher) improves the accuracy of dMRI-derived axonal orientation estimates, and may have a greater impact than high angular resolution or ultra-high $b$-values [15]. That work utilized small human samples that were scanned in a small-bore MRI scanner. The coil presented here paves the way for sub-mm resolution ex vivo dMRI on whole human brains at the high $b$-values accessible on the $3 \mathrm{~T}$ Connectome scanner. This capability will allow us to map the connectional anatomy and microstructure of the human brain at unprecedented resolutions, as well as provide reference data for evaluating in vivo dMRI scans to gain deeper insight into human brain structure at multiple scales. We expect this novel coil design, in combination with the current $3 \mathrm{~T}$ Connectome scanner equipped with $300 \mathrm{mT} \mathrm{m}^{-1}$ gradient strengths and next-generation gradient system planned for the Connectome 2.0 project [52], to advance our understanding of human brain circuitry in health and disease.

\section{Conclusion}

A 48ch close-fitting receive array coil for dMRI of whole ex vivo human brains at $3 \mathrm{~T}$ was designed, constructed, and tested with a brain-shaped phantom and an ex vivo brain. We characterized the coil with unloaded-to-loaded $Q$-ratio, noise correlation, SNR, SMS G-factor and stability measurements in comparison to a $64 \mathrm{ch}$ whole-head in vivo coil. Compared to in vivo array coils, smaller loop sizes can be used for ex vivo brain samples due to increased loading characteristics of the fixed brain tissue. This allows the design of high-channel count arrays, improving both peripheral SNR and encoding performance for accelerated imaging. Due to the high SNR and parallelism, the designed coil is well-suited for high-resolution, high $b$-value ex vivo dMRI acquisitions. 


\section{References}

[1] M. Lagana, M. Rovaris, A. Ceccarelli, C. Venturelli, S. Marini, G. Baselli, Dti parameter optimisation for acquisition at $1.5 \mathrm{t}$ : Snr analysis and clinical application, Computational intelligence and neuroscience 2010 (2010) 8. doi:10.1155/2010/254032.

[2] S. Mori, J. Zhang, Principles of diffusion tensor imaging and its applications to basic neuroscience research, Neuron 51 (5) (2006) 527-539. doi:10.1016/j.neuron.2006.08.012

[3] J. A. McNab, S. Jbabdi, S. C. Deoni, G. Douaud, T. E. Behrens, K. L. Miller, High resolution diffusion-weighted imaging in fixed human brain using diffusion-weighted steady state free precession, Neuroimage 46 (3) (2009) 775-785. doi:10.1016/j.neuroimage.2009.01.008.

[4] H. Okano, P. Mitra, Brain-mapping projects using the common marmoset, Neuroscience research 93 (2015) 3-7. doi:10.1016/j.neures.2014.08.014.

[5] T. E. Conturo, N. F. Lori, T. S. Cull, E. Akbudak, A. Z. Snyder, J. S. Shimony, R. C. McKinstry, H. Burton, M. E. Raichle, Tracking neuronal fiber pathways in the living human brain, Proceedings of the National Academy of Sciences 96 (18) (1999) 10422-10427. doi: $10.1073 /$ pnas.96.18.10422

[6] P. J. Basser, J. Mattiello, D. LeBihan, Mr diffusion tensor spectroscopy and imaging, Biophysical journal 66 (1) (1994) 259-267. doi:10.1016/S0006-3495(94)80775-1.

口 [7] H. Zeng, Mesoscale connectomics, Current opinion in neurobiology 50 (2018) 154-162. doi: $10.1016 / j . c o n b .2018 .03 .003$

[8] A. Roebroeck, K. L. Miller, M. Aggarwal, Ex vivo diffusion mri of the human brain: Technical 415 challenges and recent advances, NMR in Biomedicine 32 (2019) e3941. doi:https://doi. org/10.1002/nbm.3941.

[9] J. Beaujoin, N. Palomero-Gallagher, F. Boumezbeur, M. Axer, J. Bernard, F. Poupon, D. Schmitz, J.-F. Mangin, C. Poupon, Post-mortem inference of the human hippocampal connectivity and microstructure using ultra-high field diffusion mri at $11.7 \mathrm{t}$, Brain Structure and Function 223 (5) (2018) 2157-2179. doi:10.1007/s00429-018-1617-1.

[10] M. Modo, T. K. Hitchens, J. R. Liu, R. M. Richardson, Detection of aberrant hippocampal mossy fiber connections: ex vivo mesoscale diffusion mri and microtractography with histological validation in a patient with uncontrolled temporal lobe epilepsy, Human brain mapping 37 (2) (2016) 780-795. doi:10.1002/hbm.23066 
425

[

[11] K. L. Miller, C. J. Stagg, G. Douaud, S. Jbabdi, S. M. Smith, T. E. Behrens, M. Jenkinson, S. A. Chance, M. M. Esiri, N. L. Voets, et al., Diffusion imaging of whole, post-mortem human brains on a clinical mri scanner, Neuroimage 57 (1) (2011) 167-181. doi:10.1016/j. neuroimage.2011.03.070.

[12] J. C. Augustinack, K. Helmer, K. E. Huber, S. Kakunoori, L. Zöllei, B. Fischl, Direct visualization of the perforant pathway in the human brain with ex vivo diffusion tensor imaging, Frontiers in human neuroscience 4 (2010) 42. doi:10.3389/fnhum.2010.00042.

[13] F. Fritz, S. Sengupta, R. Harms, D. Tse, B. Poser, A. Roebroeck, Ultra-high resolution and multi-shell diffusion mri of intact ex vivo human brains using kt-dsteam at $9.4 \mathrm{t}$, Neuroimage 202 (2019) 116087. doi:10.1016/j.neuroimage.2019.116087.

[14] J. Mollink, M. Kleinnijenhuis, A.-M. van Cappellen van Walsum, S. N. Sotiropoulos, M. Cottaar, C. Mirfin, M. P. Heinrich, M. Jenkinson, M. Pallebage-Gamarallage, O. Ansorge, S. Jbabdi, K. L. Miller, Evaluating fibre orientation dispersion in white matter: Comparison of diffusion mri, histology and polarized light imaging, NeuroImage 157 (2017) 561 - 574. doi:https://doi.org/10.1016/j.neuroimage.2017.06.001. URL http://www.sciencedirect.com/science/article/pii/S1053811917304706

[15] R. Jones, G. Grisot, J. Augustinack, C. Magnain, D. A. Boas, B. Fischl, H. Wang, A. Yendiki, 1. Insight into the fundamental trade-offs of diffusion mri from polarization-sensitive optical coa herence tomography in ex vivo human brain, NeuroImage 214 (2020) 116704. doi:https: //doi.org/10.1016/j.neuroimage.2020.116704. URL http://www.sciencedirect.com/science/article/pii/S1053811920301919

[16] M. Pallebage-Gamarallage, S. Foxley, R. A. Menke, I. N. Huszar, M. Jenkinson, B. C. Tendler, C. Wang, S. Jbabdi, M. R. Turner, K. L. Miller, et al., Dissecting the pathobiology of altered mri signal in amyotrophic lateral sclerosis: A post mortem whole brain sampling strategy for the integration of ultra-high-field mri and quantitative neuropathology, BMC neuroscience 19 (1) (2018) 11. doi:10.1186/s12868-018-0416-1.

[17] B. R. Plantinga, A. Roebroeck, V. G. Kemper, K. Uludağ, M. Melse, J. Mai, M. L. Kuijf, A. Herrler, A. Jahanshahi, B. M. ter Haar Romeny, et al., Ultra-high field mri post mortem structural connectivity of the human subthalamic nucleus, substantia nigra, and globus pallidus, Frontiers in neuroanatomy 10 (2016) 66. doi:10.3389/fnana.2016.00066.

[18] J. C. Augustinack, K. Helmer, K. E. Huber, S. Kakunoori, L. Zöllei, B. Fischl, Direct visualization of the perforant pathway in the human brain with ex vivo diffusion tensor imaging, Frontiers in human neuroscience 4 (2010) 42. doi:10.3389/fnhum.2010.00042. 
[19] F. Calamante, J.-D. Tournier, N. D. Kurniawan, Z. Yang, E. Gyengesi, G. J. Galloway, D. C. Reutens, A. Connelly, Super-resolution track-density imaging studies of mouse brain: comparison to histology, Neuroimage 59 (1) (2012) 286-296. doi:10.1016/j.neuroimage.2011.07. 014

[20] J. A. McNab, B. L. Edlow, T. Witzel, S. Y. Huang, H. Bhat, K. Heberlein, T. Feiweier, K. Liu, B. Keil, J. Cohen-Adad, et al., The human connectome project and beyond: initial applications of $300 \mathrm{mt} / \mathrm{m}$ gradients, Neuroimage 80 (2013) 234-245. doi:10.1016/j.neuroimage.2013. 05.074 .

[21] K. Setsompop, R. Kimmlingen, E. Eberlein, T. Witzel, J. Cohen-Adad, J. A. McNab, B. Keil, M. D. Tisdall, P. Hoecht, P. Dietz, et al., Pushing the limits of in vivo diffusion mri for the human connectome project, Neuroimage 80 (2013) 220-233. doi:10.1016/j.neuroimage. 2013.05 .078 .

[22] C. Eichner, M. Paquette, T. Mildner, T. Schlumm, K. Pléh, L. Samuni, C. Crockford, R. M. Wittig, C. Jäger, H. E. Möller, A. D. Friederici, A. Anwander, Increased sensitivity and signalto-noise ratio in diffusion-weighted mri using multi-echo acquisitions, NeuroImage 221 (2020) 117172. doi:https://doi.org/10.1016/j.neuroimage.2020.117172 URL http://www.sciencedirect.com/science/article/pii/S1053811920306583

[23] A. Roebroeck, S. Sengupta, M. Bastiani, S. Schillak, B. Tramm, M. Waks, A. Lataster, A. Herrler, D. Tse, B. Poser, High resolution mri neuroanatomy of the whole human brain post mortem with a specialized 9.4 t rf-coil, Proc. OHBMdoi:10.13140/RG.2.2.21380.07041.

[24] S. Sengupta, F. Fritz, R. Harms, S. Hildebrand, D. Tse, B. A. Poser, R. Goebel, A. Roebroeck, High resolution anatomical and quantitative mri of the entire human occipital lobe ex vivo at 9.4 t, Neuroimage 168 (2018) 162-171. doi:10.1016/j.neuroimage.2017.03.039.

[25] B. L. Edlow, A. Mareyam, A. Horn, J. R. Polimeni, T. Witzel, M. D. Tisdall, J. C. Augustinack, J. P. Stockmann, B. R. Diamond, A. Stevens, et al., 7 tesla mri of the ex vivo human brain at 100 micron resolution, Scientific data 6 (1) (2019) 1-10. doi:10.1038/s41597-019-0254-8.

[26] A. Scholz, M. May, R. Etzel, M. Mahmutovic, N. Kutscha, L. L. Wald, A. Yendiki, B. Keil, A 48-channel ex vivo brain array coil for diffusion-weighted mri at 3t, in: Proceedings of the 27th Annual Meeting of ISMRM, Montréal, 2019, p. 1494.

[27] G. C. Wiggins, C. Triantafyllou, A. Potthast, A. Reykowski, M. Nittka, L. Wald, 32-channel 3 tesla receive-only phased-array head coil with soccer-ball element geometry, Magnetic Resonance in Medicine: An Official Journal of the International Society for Magnetic Resonance in Medicine 56 (1) (2006) 216-223. doi:10.1002/mrm.20925. 
[28] P. B. Roemer, W. A. Edelstein, C. E. Hayes, S. P. Souza, O. M. Mueller, The nmr phased array, Magnetic resonance in medicine 16 (2) (1990) 192-225. doi:10.1002/mrm.1910160203.

[29] A. Kumar, W. A. Edelstein, P. A. Bottomley, Noise figure limits for circular loop mr coils, Magnetic Resonance in Medicine 61 (5) (2009) 1201-1209. doi:10.1002/mrm.21948. URL https://onlinelibrary.wiley.com/doi/abs/10.1002/mrm.21948

[30] B. Keil, L. L. Wald, Massively parallel mri detector arrays, Journal of magnetic resonance 229 (2013) 75-89. doi:10.1016/j.jmr.2013.02.001.

[31] A. Reykowski, S. M. Wright, J. R. Porter, Design of matching networks for low noise preamplifiers, Magnetic resonance in medicine 33 (6) (1995) 848-852. doi:10.1002/mrm.1910330617.

[32] W. Edelstein, C. Hardy, O. Mueller, Electronic decoupling of surface-coil receivers for $\mathrm{nmr}$ imaging and spectroscopy, Journal of Magnetic Resonance (1969) 67 (1) (1986) 156-161. doi: 10.1016/0022-2364(86)90421-X.

[33] D. M. Peterson, B. L. Beck, G. R. Duensing, J. R. Fitzsimmons, Common mode signal rejection methods for mri: reduction of cable shield currents for high static magnetic field systems, Concepts in Magnetic Resonance Part B: Magnetic Resonance Engineering: An Educational Journal 19 (1) (2003) 1-8. doi:10.1002/cmr.b.10090.

[34] D. Hoult, The nmr receiver: a description and analysis of design, Progress in Nuclear Magnetic Resonance Spectroscopy 12 (1) (1978) 41-77. doi:10.1016/0079-6565(78)80002-8.

[35] C. Ianniello, J. A. de Zwart, Q. Duan, C. M. Deniz, L. Alon, J.-S. Lee, R. Lattanzi, R. Brown, Synthesized tissue-equivalent dielectric phantoms using salt and polyvinylpyrrolidone solutions, Magnetic resonance in medicine 80 (1) (2018) 413-419. doi:10.1002/mrm. 27005

[36] P. Kellman, E. R. McVeigh, Image reconstruction in snr units: a general method for snr measurement, Magnetic resonance in medicine 54 (6) (2005) 1439-1447. doi:10.1002/mrm. 20713

[37] K. P. Pruessmann, M. Weiger, M. B. Scheidegger, P. Boesiger, Sense: sensitivity encoding for fast mri, Magnetic resonance in medicine 42 (5) (1999) 952-962. doi:10.1002/(SICI) 1522-2594(199911) 42:5<952: :AID-MRM16>3.0.C0;2-S

[38] D. J. Larkman, J. V. Hajnal, A. H. Herlihy, G. A. Coutts, I. R. Young, G. Ehnholm, Use of multicoil arrays for separation of signal from multiple slices simultaneously excited, Journal of Magnetic Resonance Imaging: An Official Journal of the International Society for Magnetic Resonance in Medicine 13 (2) (2001) 313-317. doi:10.1002/1522-2586(200102)13:2<313: : AID-JMRI1045>3.0.CD;2-W. 
[39] K. Setsompop, B. A. Gagoski, J. R. Polimeni, T. Witzel, V. J. Wedeen, L. L. Wald, Blippedcontrolled aliasing in parallel imaging for simultaneous multislice echo planar imaging with reduced g-factor penalty, Magnetic resonance in medicine 67 (5) (2012) 1210-1224. doi: $10.1002 / \mathrm{mrm} .23097$.

[40] D. A. Feinberg, S. Moeller, S. M. Smith, E. Auerbach, S. Ramanna, M. F. Glasser, K. L. Miller, K. Ugurbil, E. Yacoub, Multiplexed echo planar imaging for sub-second whole brain fmri and fast diffusion imaging, PloS one 5 (12). doi:10.1371/journal.pone.0015710.

[41] B. Keil, J. N. Blau, S. Biber, P. Hoecht, V. Tountcheva, K. Setsompop, C. Triantafyllou, L. L. Wald, A 64-channel 3t array coil for accelerated brain mri, Magnetic resonance in medicine 70 (1) (2013) 248-258. doi:10.1002/mrm.24427

[42] R. M. Weisskoff, Simple measurement of scanner stability for functional nmr imaging of activation in the brain, Magnetic resonance in medicine 36 (4) (1996) 643-645. doi:10.1002/ mrm.1910360422

[43] J. L. Andersson, S. N. Sotiropoulos, An integrated approach to correction for off-resonance effects and subject movement in diffusion mr imaging, NeuroImage 125 (2016) 1063 - 1078. doi:https://doi.org/10.1016/j.neuroimage.2015.10.019 URL http://www.sciencedirect.com/science/article/pii/S1053811915009209

[44] B. Keil, V. Alagappan, A. Mareyam, J. A. McNab, K. Fujimoto, V. Tountcheva, C. Triantafyllou, D. D. Dilks, N. Kanwisher, W. Lin, et al., Size-optimized 32-channel brain arrays for 3 t pediatric imaging, Magnetic Resonance in Medicine 66 (6) (2011) 1777-1787. doi:10.1002/mrm.22961.

[45] T. Janssens, B. Keil, R. Farivar, J. A. McNab, J. R. Polimeni, A. Gerits, J. Arsenault, L. L. Wald, W. Vanduffel, An implanted 8-channel array coil for high-resolution macaque mri at 3 t, Neuroimage 62 (3) (2012) 1529-1536. doi:10.1016/j.neuroimage.2012.05.028

[46] F. Wiesinger, N. De Zanche, K. Pruessmann, Approaching ultimate snr with finite coil arrays, in: Proceedings of the 13th Annual Meeting of ISMRM, 2005, p. 672.

[47] A. S. Shatil, M. N. Uddin, K. M. Matsuda, C. R. Figley, Quantitative ex vivo mri changes due to progressive formalin fixation in whole human brain specimens: longitudinal characterization of diffusion, relaxometry, and myelin water fraction measurements at $3 \mathrm{t}$, Frontiers in medicine 5 (2018) 31. doi:10.3389/fmed.2018.00031.

[48] J. E. Iglesias, R. Insausti, G. Lerma-Usabiaga, M. Bocchetta, K. Van Leemput, D. N. Greve, A. Van der Kouwe, B. Fischl, C. Caballero-Gaudes, P. M. Paz-Alonso, et al., A probabilistic 

314-326. doi:10.1016/j.neuroimage.2018.08.012

[49] A. S. Shatil, K. M. Matsuda, C. R. Figley, A method for whole brain ex vivo magnetic resonance imaging with minimal susceptibility artifacts, Frontiers in neurology 7 (2016) 208. doi:10. 3389/fneur.2016.00208.

[50] S. Y. Huang, Q. Tian, Q. Fan, T. Witzel, B. Wichtmann, J. A. McNab, J. D. Bireley, N. Machado, E. C. Klawiter, C. Mekkaoui, L. L. Wald, A. Nummenmaa, High-gradient diffusion mri reveals distinct estimates of axon diameter index within different white matter tracts in the in vivo human brain, Brain Structure and Function 225 (2020) 1277-1291. doi:10.1007/s00429-019-01961-2.

[51] K. Setsompop, Q. Fan, J. Stockmann, B. Bilgic, S. Huang, S. Cauley, A. Nummenmaa, F. Wang, Y. Rathi, T. Witzel, W. LL, High-resolution in vivo diffusion imaging of the human brain with generalized slice dithered enhanced resolution: Simultaneous multislice (gslidersms), Magnetic Resonance in Medicine (2018) 141-151doi:10.1002/mrm. 26653

[52] A. Yendiki, T. Witzel, S. Y. Huang, Connectome 2.0: Cutting-edge hardware ushers in new opportunities for computational diffusion mri, in: E. Bonet-Carne, J. Hutter, M. Palombo, M. Pizzolato, F. Sepehrband, F. Zhang (Eds.), Computational Diffusion MRI, Springer, Switzerland, 2020, pp. 3-12. doi:10.1007/978-3-030-52893-5_1. 